\title{
Percutaneous approach with AngioVac system for complicated hepatic rupture in pregnancy.
}

\author{
Vincenzo Tarzia ${ }^{1}$, Antonio Piperata ${ }^{2}$, roberto vezzaro ${ }^{2}$, alessandro volpe ${ }^{2}$, and Gino \\ Gerosa $^{2}$ \\ ${ }^{1}$ Division of Cardiac Surgery, University of Padua \\ ${ }^{2}$ Affiliation not available
}

February 8, 2021

\begin{abstract}
ABSTRACT The hepatic rupture durig pregnancy is a catastrophic clinical issue associated with high fetal and maternal mortality. We describe the case of hepatic hemangioma rupture complicated by massive thrombosis of the inferior vena cava successfully treated by using AngioVac aspiration system. Although an aggressive surgical strategy is recommended, a multidisciplinary approach using modern devices can represent a new frontier in the treatment of these patients.
\end{abstract}

\section{INTRODUCTION}

The hepatic rupture is a catastrophic clinical issue that mostly occurs in cases of benign and malignant liver disease. During pregnancy, HELLP (Haemolysis, Elevated Liver enzymes, Low Platelets) syndrome or preeclampsia are major risk factors of hepatic rupture associated with high fetal and maternal mortality.

Although an aggressive surgical strategy is recommended, a multidisciplinary approach using modern devices can represent a new frontier in the treatment of these patients.

\section{CASE REPORT}

We describe the case of a 33year-old woman affected by an undiagnosed hepatic hemangioma during pregnancy. She never had health problems but, a few hours after giving birth, she presented a brutal abdominal pain and dyspnea. Her physical examination revealed no fever, normal blood pressure and sinusal tachycardia. She was agitated with cold sweating, respiratory alkalosis, and hepatomegaly. The blood tests and coagulation panel were within normal limits.

The abdominal echo-fast showed the presence of blood in the peritoneal cavity and a total body CT-scan showed a rupture of intra-parenchymal mass with a massive intra-hepatic hematoma (Figure 1) and active spreading of contrast from the right branch of the hepatic artery. Severe hemoperitoneum and some spots of pulmonary embolization were also reported. In particular, the increased abdominal pressure had been so intense to cause compression of the inferior vena cava, leading to an almost total thrombosis affecting the inferior vena cava and iliac arteries (Figure 2).

The patient was then fully monitored and transferred in emergency status to the radiological operating room. Four units of red blood cells were administered to maintain a hemoglobin level $>10 \mathrm{~g} / \mathrm{dl}$, and stable hemodynamic control was assured through low doses of inotropes during perioperative phases.

Under local anesthesia, catheterization and embolization of selective hepatic arteries were performed through the right femoral artery. The post procedural control confirmed a massive thrombotic obstruction that extended from the iliac veins to the whole inferior vena cava. 
Considering the hemodynamic stability and satisfactory control of bleeding, we decided to perform an aspiration of the iliocaval thrombus with the use of AngioVac system (Figure 3). After the administration of 5000UI of heparin, the right femoral vein was cannulated in a percutaneous standard fashion and the 22French AngioVac inflow cannula was advanced through the sheath of a 26-French GORE DrySeal (W.L. Gore \& Associates, Inc, Newark, DE) into the inferior vena cava. The reinfusion cannula was inserted through the right jugular vein and the lines were connected to the circuit (Figure 3). Once the veno-venous bypass was established, the complete extraction procedure was performed using suction power (Figure 4). The success of the procedure was defined by the complete aspiration of the thrombus, checked by the return of an antegrade flow into the vena cava, at the angiographic control (Figure 5A). The procedure was then completed with the placement of a caval-filter, to avoid any other small embolism (Figure 5B). No complications were reported, and the CT-scan control showed a good result of both procedures, with satisfactory control of bleeding and venous flow into the inferior vena cava.

Three days later, thanks to the stable condition, we decided to complete the treatment with a peritoneal wash out with laparoscopic approach, without complications. The total ICU and hospitalization time was 9 and 34 days, respectively. No pulmonary embolism or other intra-abdominal bleeding episodes were reported.

Up to now, total follow up time is 3 years and 3 months and no complications occurred.

\section{DISCUSSION}

The spontaneous hepatic rupture is a tragic condition and is mostly encountered in cases of benign and malignant liver diseases [1]. During pregnancy, HELLP syndrome and preeclampsia are a major risk factor of hepatic rupture, associated with about $40 \%$ of mortality. The risk persists even after delivery.

Delayed diagnosis is probably the major contributing factor for high perinatal (44\%) and maternal mortality rate ranging from $7.5 \%(2)$ to $39 \%[3]$.

If there is a strong clinical suspicion of liver rupture, laparotomy should be practiced immediately to reduce mortality. The surgical procedures that can be performed range from hepatic artery ligation or embolization to liver packing, omentum patches or, intra-abdominal perihepatic vacuum assisted closure (VAC) therapy. In addition, if extensive hemorrhagic necroses lead to progressive hepatic failure, a liver transplant should be considered. It is worth mentioning that despite surgical efforts, about one-third of the patients with liver rupture die in hemorrhagic shock.

In this described case, the challenge was not only the management of the hepatic rupture but also the unexpected complication of ilio-caval thrombosis.

A multidisciplinary approach was employed to minimize the risks and ensure the best result for the patient. In this context, a new and extremely versatile tool available for the cardiovascular surgery team at our hospital was used to avoid otherwise invasive intervention. The AngioVac (AngioDynamics,Lathan, New York) aspiration system consists of a $22 \mathrm{~F}$ suction cannula, an extracorporeal circuit with a filter, and a reinfusion catheter for returning blood to the patient (Figure 3). This device can drain a large volume of blood $(>3 \mathrm{~L} / \mathrm{min}$ ) allowing to remove clots, vegetation, or other debris. It is a safe and easy-to-use tool and the results reported in literature are very encouraging [4].

In this case, AngioVac was considered the first line strategy to carry out, avoiding open surgery, general anesthesia, and all related risks.

More than 3 years since the event, the patient is in excellent health condition, the caval filter has been removed and the last CTscan control shows a marked reduction in liver mass to $>50 \%$ of the initial size.

Our case highlights that liver rupture and subsequent massive thrombosis of inferior vena cava could be dangerous and difficult to manage for several reasons. Firstly, the high risk related to massive pulmonary embolism. Secondly, the risk of reduced venous drainage in splanchnic organs. Thirdly, for the high bleeding risk due to thrombolysis treatment. Lastly, technical challenge in inferior vena cava filter implantation. 
For these reasons, we believe that technical and technological evolution made it possible to manage a potentially fatal complication by avoiding open surgery and major organ manipulation by using a multidisciplinary approach and the best devices available in each medical specialty [5].

This strategy allowed, to avoid laparotomy, general anesthesia, and to reduce the risk of infection.

To the best of our knowledge, this is the first reported case of liver rupture complicated by massive ilio-caval thrombosis that was successfully managed with a minimally invasive approach, without complications.

\section{Author contribution:}

Vincenzo Tarzia: conceptualization, project administration

Antonio Piperata: conceptualization, writing

Roberto Vezzaro: project administration

Alessandro Volpe: project administration

Gino Gerosa: Critical revision of article, Approval of article, supervision.

\section{References}

1. Chen ZY1, Qi QH, Dong ZL. Etiology and management of hemorrhage in spontaneous liver rupture: a report of 70 cases. World J Gastroenterol 2002; 8:1063e6.

2. Dubey S, Rani J. "Hepatic rupture in preeclampsia and HELLP syndrome: A catastrophic presentation". Taiwan J Obstet Gynecol. 2020 Sep;59(5):643-651. doi: 10.1016/j.tjog.2020.07.003. PMID: 32917311.

3. Reck T, Bussenius-Kammerer M, Ott R, Muller V, Beinder E, Hohenberger W. Surgical treatment of HELLP syndrome-associated liver rupture- an update. Eur J Obstet Gynecol Reprod Biol 2001; 99:57e65.

4. Hameed I, Lau C, Khan FM, Wingo M, Rahouma M, Leonard JR, Di Franco A, Worku BM, Salemi A, Girardi LN, Gaudino M. AngioVac for extraction of venous thromboses and endocardial vegetations: A meta-analysis. J Card Surg. 2019 Apr;34(4):170-180.

5. Gerosa G, Longinotti L, Bagozzi L, D'Onofrio A, Zanella F, Pittarello D, Tarzia V. Transapical Aspiration of a Mitral Mass With the AngioVac System on a Beating Heart. Ann Thorac Surg. 2020 Nov;110(5):e445-e447.

\section{FIGURES}

Figure 1

Title: Pre-operative CT-scan. 

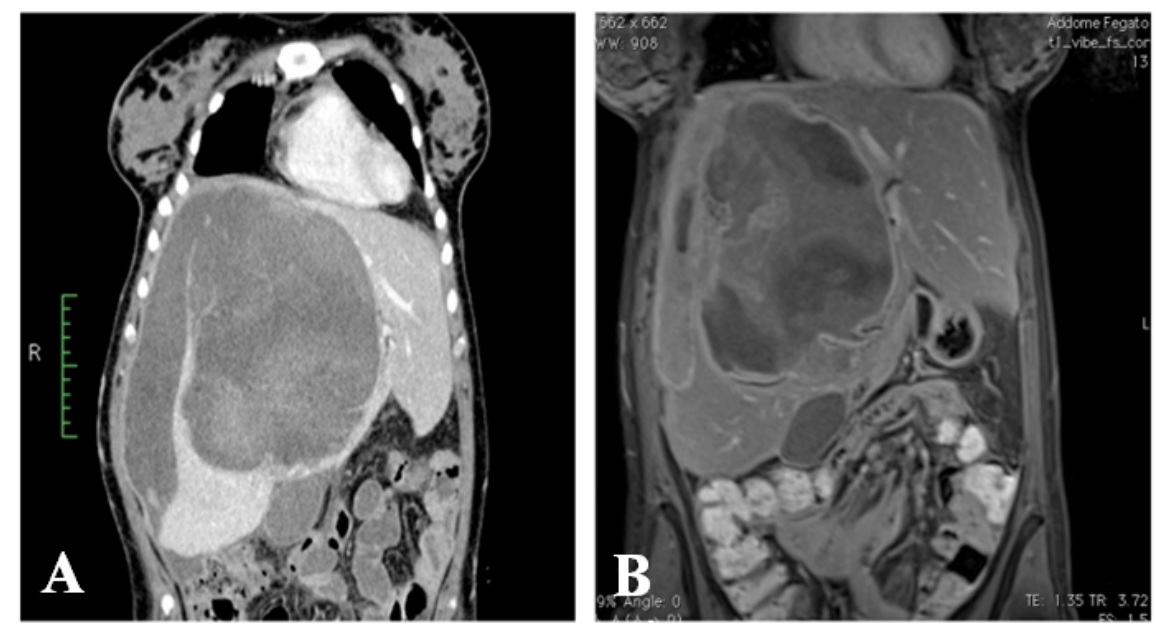

A and B: the massive intrahepatic hematoma.Figure $\mathbf{2}$

Title: Venous angiography of inferior vena cava
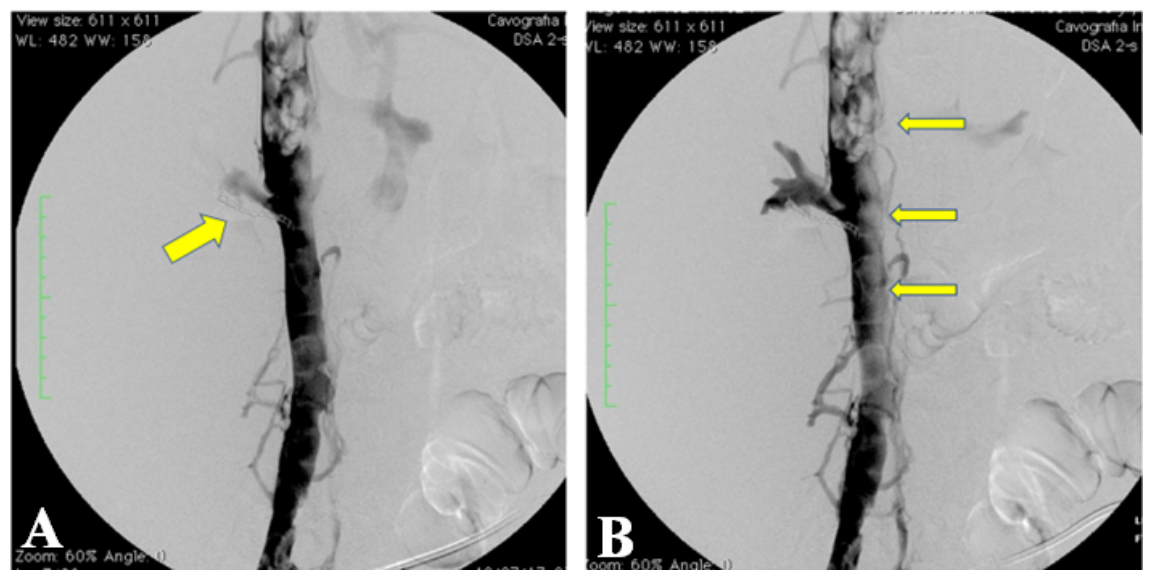

A : embolization of hepatic artery (arrow). B : Massive thrombosis of inferior vena cava.

Figure 3

Title: The AngioVac aspiration System 


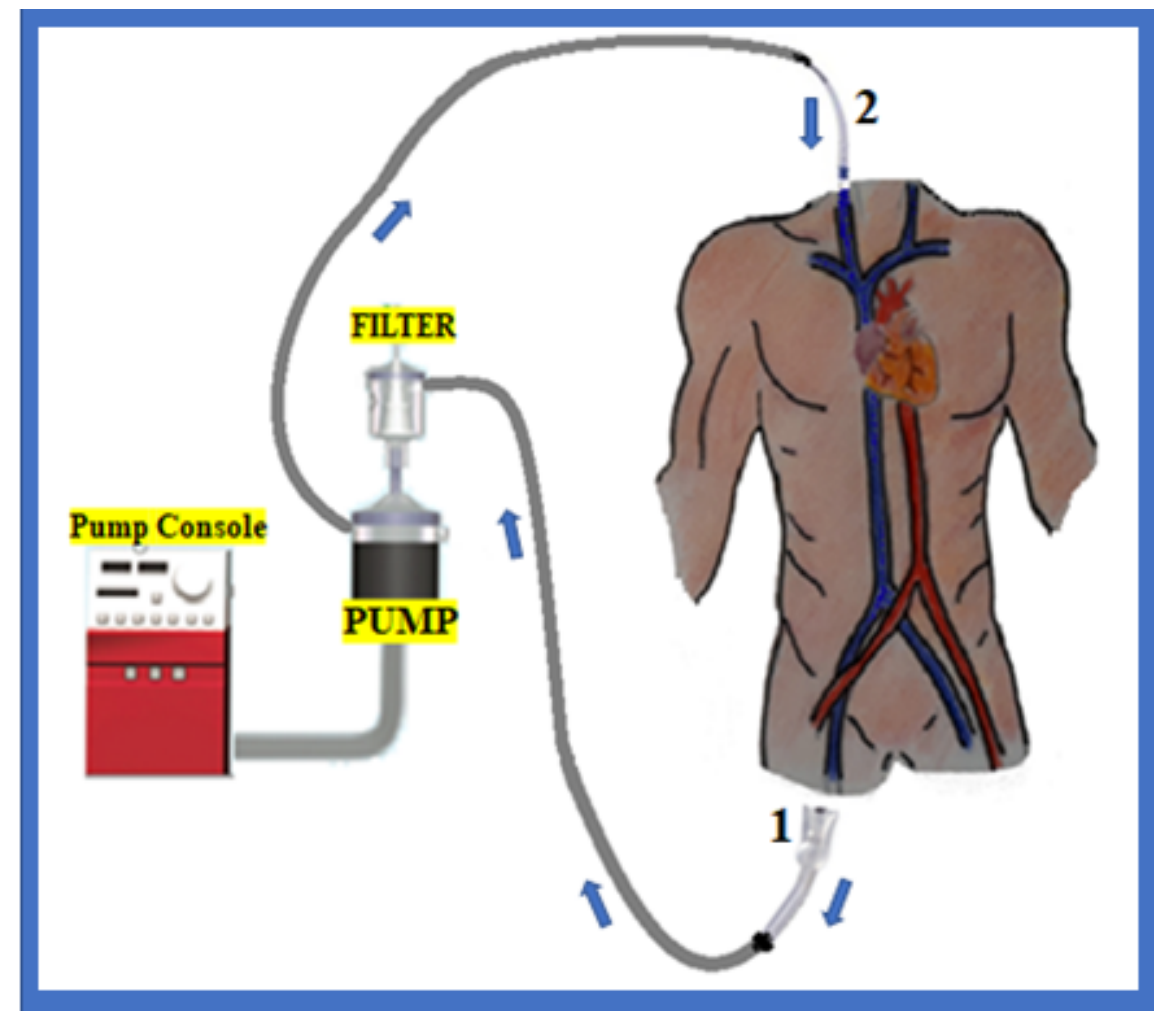

1: AngioVac cannula is inserted through the right femoral vein.2: Reinfusion cannula through the right jugular vein.

\section{Figure 4}

Title: AngioVac filter and thrombi

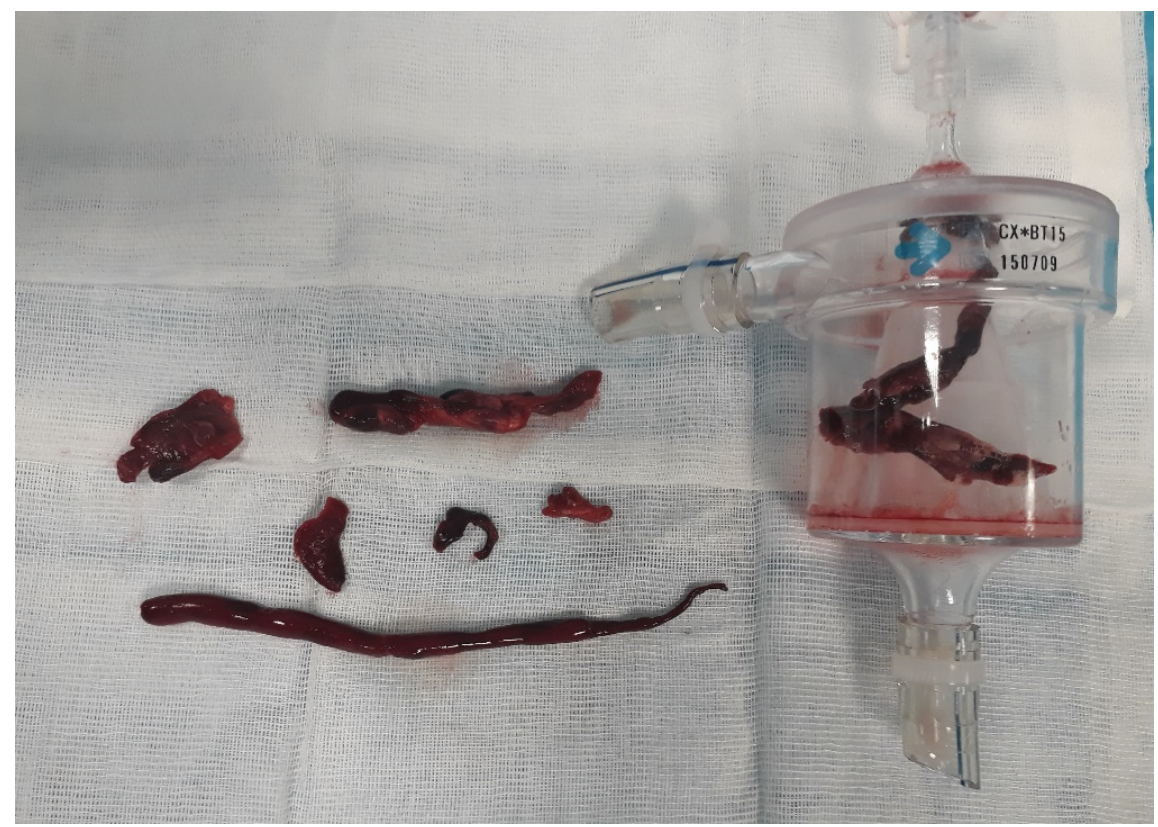


Figure 5

Title: Post-operative angiographic control
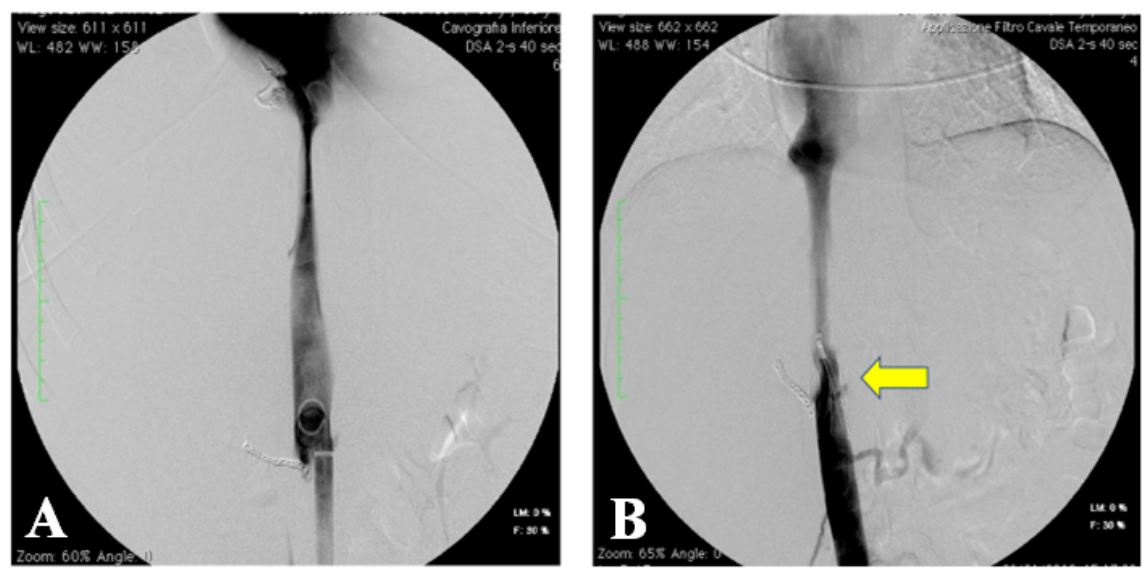

A : Angiographic control of inferior vena cava. B : positioning of caval filter (arrow)
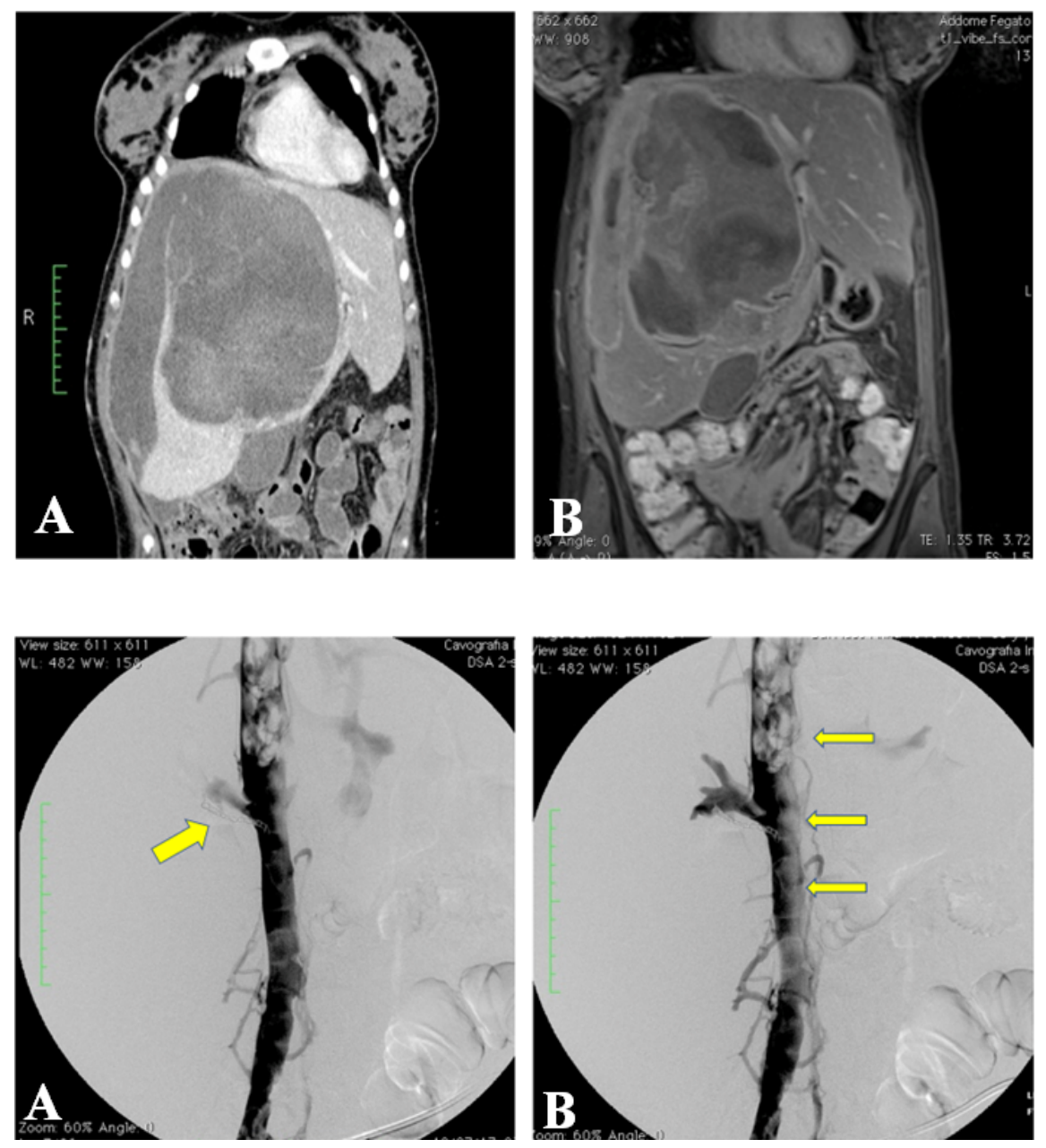

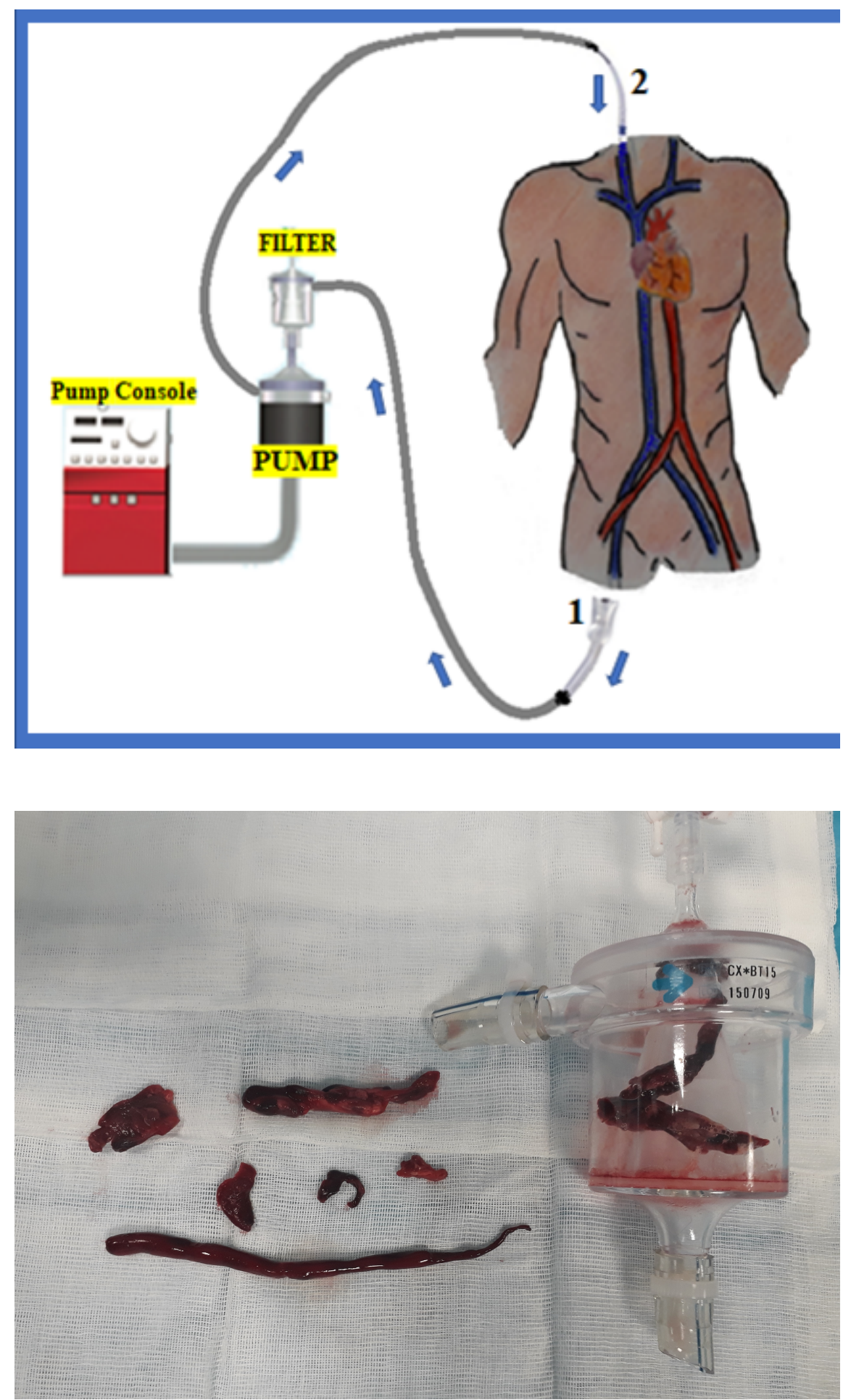


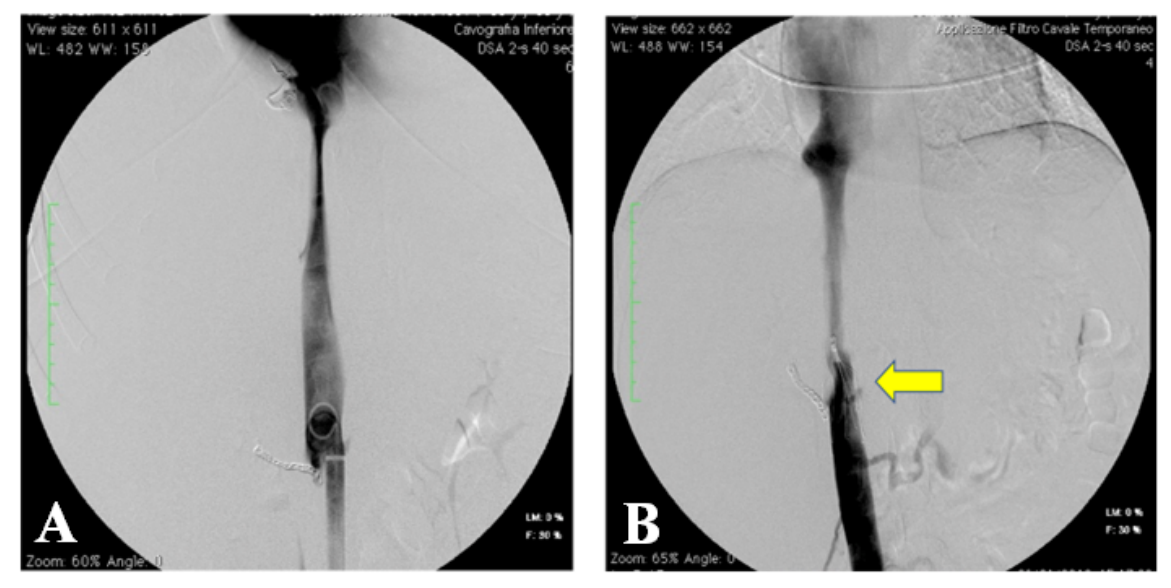

